\section{Journal homepage: http://www.journalijar.com Journal DOI: 10.21474/IJAR01}

\section{RESEARCH ARTICLE}

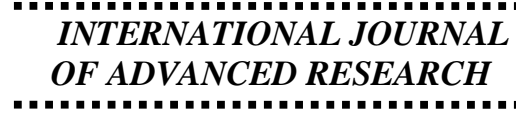

" म "

\title{
A secure reversible medical images.
}

Sukhwinder Kaur and Rajbhupinder Kaur.

PG student, Assistant Professor, Yadavindra College of Engineering and Technology, Talwandi Sabo, Punjab 151302, India.

\section{Manuscript Info}

Manuscript History:

Received: 10 May 2016

Final Accepted: 19 June 2016

Published Online: July 2016

Key words:

watermark, images, information.

*Corresponding Author

\begin{abstract}
The goal of water markings to hide the existence of the message from unauthorized party. The modern secure image watermarking presents a task of transferring the embedding information to destination without being detected by the attackers. Image watermarking adds additional information about host image in the form of logo/audio/video or text. Security and authentication is the major concern in field of medical images there are various techniques that have been used so far this paper explain various techniques and parameters for security of medical images.
\end{abstract}

Sukhwinder Kaur.

Copy Right, IJAR, 2016,. All rights reserved.

\section{Introduction:-}

Medical health care domain is more and more exploitation public network to exchange electronic patient record between hospitals and health centres[2]. In the new time edical images area unit delivered from associate assortment from calming imaging instrumentality for instance CT (computed tomography), MRI (magnetic resonance imaging), and electronic tolerant record (EPR) could also be a standout amongst the advanced design regarding the particular economical refined components. Patient, points of identification, healing center information alongside the restorative image to consistent, transmission of electronic patient records (EPR) through property right networks is risky and insecure. EPR may be intercepted during their transmission.EPR area unit is very sensitive to any reasonably modifications. minor manipulations in EPR causes wrong identification. Thus authenticity associated with security of an epr is one among the necessary issues notably once pertinent patient info is transmitted across public networks. The diagnosis wants confidentiality, accessibility and dependability, confidentiality implies that solely the initial users have access to the information [1]. Dependability relies on integrity that the data has not been changed by unauthorized persons; and authentication intends that the data belongs so to the right patient. Hospital patient management system is enforced in additional hospitals these days, designed to remodel the manual method of maintaining and accessing patient medical documents into electronic medical record (EMR) or electronic health record (EHR). EMR is used to resolve the problem of manual technique. To safeguard the personal data against unauthorized viewers, we are able to use anyone of the techniques for medical image information that are cryptography or watermarking. Telemedicine is a method by which electronic, visual and audio communications are accustomed with support practitioners at remote sites with diagnosing and consultation procedures, like remote clinical examinations and medical image transfers. Telemedicine is legally regulated by laws and constraints concerning the access of information contained in personal medical files.

\section{Difficulties of medical image security are[1] [2]:-}

1. It is very difficult to get better PSNR after extraction of watermarked data into host image.

2. Large amount of auxiliary data is required for telemedicine application. 
Following is the basic flow chart of the system for medical image secure:-

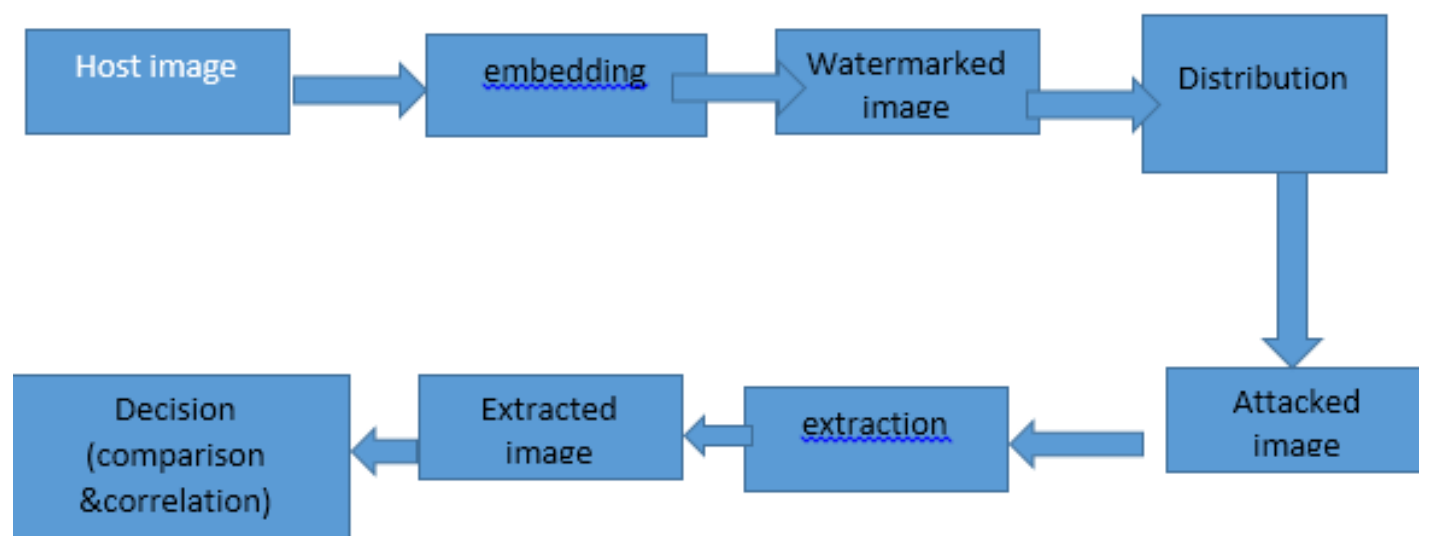

Fig1.1:- General image water marking procedure.

\section{Literature review:-}

Divecha Nidhi and Jani N.N.Dr (2013) [1]:-

Watermarking topic upheld DCT-DWT-SVD, where every region unit uses non blind techniques. One is predicated on SVD of dc coefficients abuse second level DWT deterioration and diverse is considered SVD of all DCT values of second level DWT creation of spread image. For therapeutic imaging one of the strategy is blend DCT-DWTSVD which apply one level DWT disintegration of shade picture pick 11 band for watermarking. During this dc steady essentially based watermarking subject for shading picture is normally prescribed. They apply moving edge disintegration one level to paint picture and this strategy separates the band into $4 * 4$ sub pieces and DCT is connected. To begin with DCT cost is looked over all sub squares. At that point SVD is performed there on. SVD is connected to the present network and solitary qualities range unit is changed with particular estimations of watermark, reverse SVD, opposite dct and converse DWT is performed to incite watermarked picture. This strategy is utilized for dim scale picture as secured picture and twofold or grey scale watermark.

\section{Divecha Nidhi and Jani N.N.Dr (2015) [2]:-}

The system implants information into slightest fundamental piece abuse knowledge compression. Reversible watermarking technique isutilized to support abnormal state of security of spread picture and the picture is partitioned into blocks. The upper connection is inalienable in neighboring part. This methodology is utilized to make qualification between attached point part and its neighbor pixels in vertical, even and slanting course for that piece. The qualification between neighboring pixels is utilized as a data implanting space; i.e. World top of the refinement histogram is utilized to embed watermark. Reversible watermarking strategy reestablishes unique substance when watermark expulsion from watermarked content. This might be abused to make watermarking determination for quality-delicate and security intensely mindful applications that don't empower even minor changes in substance at any phase in correspondence. This system invalidates incredible measure of overhead information or area map at collector wrap up by altering a photo component in every sub piece. Picture component inside the upper left corner of each sub piece is altered which implies that its dark cost is not adjusted all through implanting strategy. It misuses comparability between neighborhood pixels to get refinement picture with higher capacity. Histogram of those varieties is made and implanting is done in its top. Adjusted refinement is further to a set reason picture component to maintain a strategic distance from undercurrent. Procedure is flexible and may be tuned to join different crests inside the distinction histogram that enhances the data inserting capacity.

Singh menu, et al. (2013) [3]:-

Digital watermarking technique is used to incorporate hide information in transmission substance to affirm security suitability or essentially in naming application. This strategy name is advanced watermarking which is useful for stronghold of pictures, video and content at whatever point any fakers tries to harm or modify the computerized information. It will just catch on the reason of recovered watermark picture. Watermarking is a basic a piece of data covering that exists to secure copyright content into mixed media framework .when the mixed media framework 
substance is offered over the web trade of sight and sound framework content infringement issues come into the picture .Digital watermarking is required to prove authenticity of an image and verification messages or copyright notices as well to avoid forgery. Digital watermarking technique are often generally classified into 2 major classes

[3]

1. Spatial domain watermarking

2. Frequency domain watermarking

\section{Spatial domain watermarking:-}

During this watermarking copyright data is value-added by dynamic picture element values of host image and least vital bit insertion is more style of spatial domain. However such algorithms have low payload, they'll be simply discovered and quality of image when embedding the copyright data and extracted watermark isn't acceptable as element strengths square measure directly modified in these algorithms. [10]the characteristics of spatial domain watermarking techniques is that the watermark is connected in the pixel area and no changes are connected to the host signal amid watermark installing. Moreover, blend with the host sign depends on basic operations, in the pixel space. In addition to this the watermark can be identified by connecting the normal example with the got signal. The disadvantage of all the spatial area strategies is that, they modify the host picture amid installing stage. Moreover, they have the least piece limit and the most reduced imperviousness to jpeg pressure. It can survive straightforward operation, for example, editing, and any expansion of clamor. However lossy pressure is going to overcome the watermark. Once the calculation was found, it would be simple for a moderate gathering to adjust the watermark. One of the real impediments in spatial space is the limit of a picture to hold the watermark.

Any watermarking formula has 2 elements.

1. Embedding formula and

2. Extraction formula.

\section{Frequency domain [3]:-}

This technique has inserting into frequency coefficients of host image. It gives information with respect to action ability and hardness against shifted geometrical attacks. This strategy is far solid than spatial area because of the inserting watermark into the modified recurrence coefficients of changed image. Various watermarking revamp spaces are fourier redesign $(\mathrm{dft}),(\mathrm{dct})$ discrete round capacity remodel, (dwt) separate wavelet remodel, short time fourier remodel, continuous wavelet transform (cwt.), and discrete cosine transform (dct) or blend of dct and dwt.

\section{Xpeng Zhang [4]:-}

Reversible data hiding method is used to embed additional message into some twisting inadmissible cover media like as medical pictures, with a reversible way so the initial cover substance are regularly dead redesigned when extraction of the hidden message. Encryption is an effective and standard that proposes security assurance. Keeping in mind the end goal to immovably impart a mystery picture to elective person, a content proprietor could write in code the image before transmission. In this system a substance proprietor encrypts the initial uncompressed picture exploitation associate in nursing coding key to supply associate in nursing encoded picture. At that point information recognize ledgean data hider installs extra knowledge into the encoded picture utilizing an information hiding key through that doesn't know the underlying substance. While encoding the complete learning of an uncompressed picture by a steam cipher, the additional information are regularly implanted into picture by changing a modest low extent of encrypted learning with an encrypted picture containing extra information, one could first decipher it exploitation the coding key, furthermore the unscrambled variant is practically equivalent to the initial image. Basically reversible knowledge covering topic for encoded picture with low calculation multifaceted nature is purposed which comprises of picture coding, information implanting and learning extraction stages. The information of unique picture territory unit is completely scrambled by a stream cipher. The implanted knowledge is frequently appropriately separated though the underlying pictures that are regularly dead recouped. In accordance with this paper for making certain the right learning extraction furthermore the great picture recuperation, we tend to could give the piece perspective length a chance to be a giant worth, such as 32, or present blunder revision component before information action to shield the extra knowledge with the cost of payload reduction.

\section{Singal Akhil and Rajni [5]:-}

Pattern compression fractal image compression [5] could be a new technique in compression field by employing a contractive redesign that the secured focuses area unit shut thereto of unique image. This expansive field joins in itself massive quantities of committal to composing conspire, that are printed while being investigated during this field. Method depends on eliminating high recurrence parts of the sign by putting away exclusively the low 
recurrence parts (discrete cosine remodel algorithm).this strategy is utilized on jpeg (still pictures), mpeg (movement video pictures), and h.261(video telecom on isdn lines) pressure algorithms. The other procedure is example pressure. This framework tries to utilize relative repetition present inside the comm. On place pictures in order to accomplish higher pressure proportions yet as keeping up sensible picture quality. In this, the picture is part into non-covering shift squares and covering space pieces wherever the dimensions of area square are greater. At that point for each the premier comparative space piece is discovered exploitation the mean sq. Error (MSE).

Fractal image compression technique have further two techniques.

1. Fractals

2. Fractal image compression

\section{Fractals:-}

Fractal is a structure made of various examples and structures that can happen in any diverse sizes inside a picture. The term fractal was utilized by B. Mandelbrot for depicting the tedious examples, structures happening in a picture. The watched structures are fundamentally the same as each other w.r.t. Size, introduction, and turn or flip.

\section{Fractal image compression:-}

In this a photocopy machine reduces the measure of the image significantly and increases the picture by three times. This picture is called as attractor image on the grounds that any underlying picture will merge to that picture in rehashed running. This depicts the changes are contractive in nature i.e. In the event that the change is connected to two purpose of any picture, it must unite them. Fractal based coding is that the encoder is exceptionally complex. The multifaceted nature is because of the picture square preparing required for reach and area pieces. Additionally much calculation is likewise required in the mapping procedure of the extent and domain block. Along these lines, one the most communicating highlight of the fractal picture pressure is that it's deciphering procedure in extremely basic and simple. The fundamental idea in this pressure plan is to utilize the ifs to imitate pictures. By dividing any picture into [8 8], [16 16] pixels, the littler segments are recreated by fractal change. The pace up in the disentangling time by means of the utilization of the fractal picture pressure ought to be considered as a fascinating innovation. It is evitable that there are numerous applications where the fractal way of the picture can be utilized for computational purposes.

\section{S. Priya and R. Varatharajan[6]:-}

Joint watermarking combines the watermarking of the encrypted information in medicinal pictures in order to give more security using the advantages of cryptography and watermarking techniques .Joint medical picture watermarking is to encapsulate fundamental information inside a picture in vitality stuffed regions, which is advanced as for picture quality and to give a second level security by incorporation of best in class cryptographic standard. It is a zero-knowledge watermarking calculation which needs the first picture for the discovery procedure. A visually impaired watermarking calculation taking into account most extreme wavelet coefficient quantization for copyright insurance. The watermark is inserted in the neighborhood most extreme coefficient which can successfully oppose assaults. Either non-geometry or geometry assaults. The watermark can adequately oppose regular picture preparing attacks, especially by jpeg pressure (with a quality variable more prominent than 20) and gaussian noise with a variation of under.

\section{Water marking threats:-}

There are such a variety of dangers for watermarking by which this procedure needs ensured each time. As the watermarking strategies created by researches, hackers are produced 2 new techniques to assaults to decimate watermark. So each time algorithms should be heartier for counteracting attacks. [7]

The assaults by which therapeutic pictures can be influenced are:

Picture compression: Lossy pressure can bring about the devastation of an image's watermark. Geometric transformations is the turn, interpretation sheering, or resizing of a picture.

Picture upgrades : honing, shading alignment, contrast change

Picture structure: the expansion of content, winnowing with another picture, and so on. 


\section{Parameters used:-}

MSE (mean square error) [7]:-

It is estimation of the midpoints of the square of error of the blunder that is distinction amongst estimator and what is assessed. In the info picture, $\mathrm{m}, \mathrm{n}$ is the quantities of rows and columns. At the point when the parameter to estimate to be scalar, the MSE is equal to expected estimation of square of distinction between the worth taken by the estimator and the true value of the parameters. At the point when the parameters to be assessed in vector we take the euclidean known of distinction before processing the square.

$$
M S E=1 \div M N \sum_{i}^{M} \sum_{i}^{N}(W i j-H i j)^{2}
$$

The fundamental advantages of MSE is that it is anything but difficult to interrupt the working of framework and numerical examination.

PSNR (peak signal noise ratio) [7]:-

It is an engineering term for the proportion between the maximum possible force of a sign and force of corrupting noise is that effects the representation of the signal. PSNR is express term of logarithm decimal scale. In PSNR, we take square of the top worth in the picture and partitioned by it mean square error.

$$
\text { PSNR }=10 * \log \left(\mathrm{P}^{2} / \mathrm{MSE}\right)
$$

It is utilized to quantify the nature of picture after recreation. Higher the PSNR increases the reproduction.

\section{Bit Error Rate(BER)[8]:-}

The rate at which errors occur in the transmission of computerized information when information is transmitted over an information join there is possibility of errors being brought into the system so it is important to get to the execution of the framework and bit mistake rate gives in perfect route in which times can be accomplished .

\section{BER=i/PSNR:-}

BER is end to end estimation of the execution of the system including the transmitter, beneficiary medium between too. In along these lines BER empowers the real execution of a system in operation to be tried instead of testing the segment part and trusting that they will work acceptable when set up. In the event that the estimation of BER is more like zero, it implies that nature of the picture is great. Variable influencing bit blunder rate are impedance build transmitter control. Low request regulation, lesser bandwidth. It is important to adjust the entire accessible element to accomplish an acceptable piece mistake rate.

\section{Capacity[7]:-}

It is refers to the amount of mystery data can be hidden into a picture higher the limit of the picture to hide information better will be procedure the maximum measure of data that can be conveyed in the media, steganography, algorithm can be embedded without being conveyed in the media to apply unmistakable change. High limit steganography algorithm is used to assess the principle parameters.

\section{Security:-}

It alludes to how much the information is secure from the steganlysis. The security parameter is variable that measures the info size of the calculation problem, both the source necessities of cryptography calculation or convention are communicated in term of security parameters. The security parameters are generally express in unary representation. So that by the time many-sided quality of the cryptography algorithm is polynomial in size of input. 
compression of all techniques explained above:-

\begin{tabular}{|c|c|c|}
\hline Techniques & Advantages & Disadvantages \\
\hline $\operatorname{Dct}[9][10]$ & $\begin{array}{l}\text { 1.Genuine-value } \\
\text { 2.Better vitality } \\
\text { 3.Compaction coefficients are almost uncorrelated }\end{array}$ & $\begin{array}{l}\text { 1.Block wisedct destroys the invariance } \\
\text { 2.Higher frequency components } \\
\text { 3.Not work with scaling attacks }\end{array}$ \\
\hline Svd[11] & $\begin{array}{l}\text { 1.Eligible algorithmic complexity. } \\
\text { 2.A powerful technique in matrix decomposition }\end{array}$ & $\begin{array}{l}\text { 1.Unduly expensive computationally. } \\
\text { 2.Operates on a fixed matrix. }\end{array}$ \\
\hline Dwt[10] & $\begin{array}{l}\text { 1. Good localization in time, spatial frequency domain } \\
\text { 2.Higher compression ratio } \\
\text { 3.Effective for structural attacks } \\
\text { 4.Powerful for cropping. }\end{array}$ & $\begin{array}{l}\text { 1.Expense of processing might be } \\
\text { higher. } \\
\text { 2.Longer compression time. } \\
\text { 3.Noise/blur near edges of images or } \\
\text { video outlines. }\end{array}$ \\
\hline Data hiding[12] & $\begin{array}{l}\text { 1. Simplicity, } \\
\text { 2.High imperceptibility } \\
\text { 3.High capacity. }\end{array}$ & $\begin{array}{l}\text { 1.Fragile strategy } \\
\text { 2. Not tolerate any control. } \\
\text { 3.Smallest modification need for } \\
\text { destroy hidden data }\end{array}$ \\
\hline $\begin{array}{ll}\text { Fractral } & \text { image } \\
\text { compression[9] } & \end{array}$ & $\begin{array}{l}\text { 1.Faster decompression speed. } \\
\text { 2.Quick access }\end{array}$ & 1.Lengthy compression step. \\
\hline Joint[13] & $\begin{array}{l}\text { 1.More than two-party plan } \\
\text { 2.The size of watermark is autonomous of number of } \\
\text { Merchants }\end{array}$ & 1.Computationally insufficient \\
\hline Blind & $\begin{array}{l}\text { 1. Not use the original Image } \\
\text { 2.Only the secrete key is enough to extract the } \\
\text { watermark }\end{array}$ & $\begin{array}{l}\text { 1. Watermark can be extracted only } \\
\text { from watermarked } \\
\text { Image }\end{array}$ \\
\hline
\end{tabular}

\section{Conclusion:-}

In this review paper, given is explanation of different techniques and parameters on which security of medical images can be calculated. In addition to this, advantages and disadvantages of techniques are also explained. This review paper gives clear idea about basic secure medical images. This paper will be very helpful for beginners in secure reversible medical images.

\section{References:-}

1. Divecha Nidhi H. and Jani N.N. Dr (2013),"Implementation and performance analysis of DCT-DWT--SVD based watermarking algorithms for color images" international conference on intelligent systems and signal processing (ISSP), IEEE, 17july.

2. Divecha Nidhi H. and Jani N. N. Dr (2015), "Reversible watermarking technique for medical images using fixed point pixel" fifth international conference on communication systems and network technologies" ieee.

3. Singh Menu, Singhal Abishesk and Chaudhary Ankur (2013),"Digital image watermarking techniques: a survey" international journal of computer and telecommunication, volume 4, issue 6 .

4. Zhang Xinpeng (2011),"Reversible data hiding in encrypted image "signal processing letters, vol 18, no.4.

Priya1 .S1 Andvaratharajan2 R. (2015),"Embedding and extraction techniques for medical images -issues and challenges, computer science \& information technology (CS \& IT), CNDC.

5. Singal Skhil and Rajni (2014),'Performance analysis of medical image using fractal image compression, international journal of engineering research and general science volume 2, issue 4, June-July.

6. Saini Lalit Kumar, Shrivastava (2014), “Survey of digital watermarking techniques and its application" international journal of computer science trends and technology (IJCST) - volume 2 issue 3.

7. http://www.radio-electronics.com/info/rf-technology-design/ber/bit-error-rate-tutorial-definition.php (15 June 2015)

8. Kuppusamy K. Dr. (2013), “Ilackiya fractal image compression algorithmic techniques”, international journal of computer \& organization trends -volume3 issue4.

9. Monika Patel Priti Srinivas Sajja Ravi K. Sheth (2013),"Analysis and survey of digital watermarking techniques” international journal of advanced research in computer science and software engineering, Volume 3, issue 10.

10. http://www.slideshare.net/suritd/ppt1-48438386 (15 June 2015) retrieved.

11. Mishra1 Minati, Mishra Priyadarsinand Adhikary flt. Lt. Dr. M.c., "Digital image data hiding techniques: a comparative study", Fakir Mohan University, Balasore, Odisha, India.

12. Kalaivani1 I., Bhavani R.R (2015), "Watermarking for digital content- a survey" international journal of engineering and computer science ISSN: 2319-7242volume 4 issue 8, page no. 13678-13680

13. Hamed Modaghegh Hamed, Hossein Khosravi R. Hossein, Akbarzadeh R-Mohammad (2009), "A new adjustable blind watermarking based on GA and SVD, 6th international conference on innovations in information technology. 